\title{
A GMSK VHF-Uplink/UHF-Downlink Transceiver for the CubeSat Missions
}

\section{Thermo-Functional Performance}

\author{
Yaseen Zaidi • Robert Ryk van Zyl • Norman G. Fitz-Coy
}

Received: date / Accepted: date

\begin{abstract}
Functional and thermal performance characteristics of a Very High Frequency/Ultra High Frequency (VHF/UHF) transceiver based on Gaussian Minimum Shift Keying (GMSK) modulation are presented. The transceiver has been designed for CubeSats telemetry and commanding needs or low rate data download. The design is validated at $27 \mathrm{dBm}, 30 \mathrm{dBm}$ and $33 \mathrm{dBm}$ transmitting powers over $-20^{\circ} \mathrm{C}$ to $+51^{\circ} \mathrm{C}$. Under these thermal conditions, the transmitter spurious dynamic response shows little if any change and the average sensitivity of receiver at the $12 \mathrm{~dB}$ SIgnal Noise And Distortion (SINAD) is $-116.7 \mathrm{dBm}$ at $140 \mathrm{MHz}$ and $-116.78 \mathrm{dBm}$ at $149.98 \mathrm{MHz}$. The transmitter and receiver frequencies are stable and the current consumption as well the output RF levels are steady. The design has been verified against a simulation model which allows system tradeoff analysis. The measurements demonstrate the transceiver made with commercial grade parts has dependable performance at the low earth altitudes and orbital heating conditions.
\end{abstract}

Keywords CubeSat · GMSK · SFDR · SINAD . Thermal $\cdot$ UHF and VHF

Y. Zaidi (四) - R. R. v. Zyl

yaseen.zaidi@ieee.org

French South African Institute of Technology

Cape Peninsula University of Technology

Bellville 7530, South Africa

N. G. Fitz-Coy

Dept. of Mechanical \& Aerospace Engineering

University of Florida

Gainesville, FL 32611, USA

\section{Introduction}

The Very High Frequency/Ultra High Frequency (VHF/UHF) is widely recognized as a model of authority for excellence in performance in space communications. The technology has distinguished itself over several decades as reliable in the high-end missions [1] [2]. The technology also rules as the standard communications fundament in the missions flying the CubeSat class of satellites [3] [4]. Emerged from the terrestrial mobile radio applications, Gaussian Minimum Shift Keying (GMSK) [5] [6] is a type of fast Frequency Shift Keying (FSK). It, too, has settled as a robust, spectrum compact, continuous phase but constant envelope modulation scheme, suited for noisy environments and requiring nonlinear Class $\mathrm{C}$ amplification. In this paper, we examine, through functional and temperature measurements, the several performance metrics of a GMSK based VHF/UHF communications subsystem that has been successfully flying the ZA-CUBE-1 mission in full duplex mode and its simplex variant will be flying the ZA-CUBE-2.

\section{VHF/UHF Transceiver}

VHF/UHF transceiver (Fig. 1) is compatible with the CubeSat nanosatellite standard and CubeSat Kit $\mathrm{PC} / 104$ form factor. The frequency of RX/TX operation is software selectable within the band and is adjustable in channel bandwidth steps. Additionally, a Consultative Committee for Space Data Systems (CCSDS) 1/2 rate convolutional encoder with constraint length $K=7$ (171 octal, 133 octal) can be enabled to encode the data. The commercial band transceiver has the features listed in Table 1. The AFSK does not operate in full duplex 

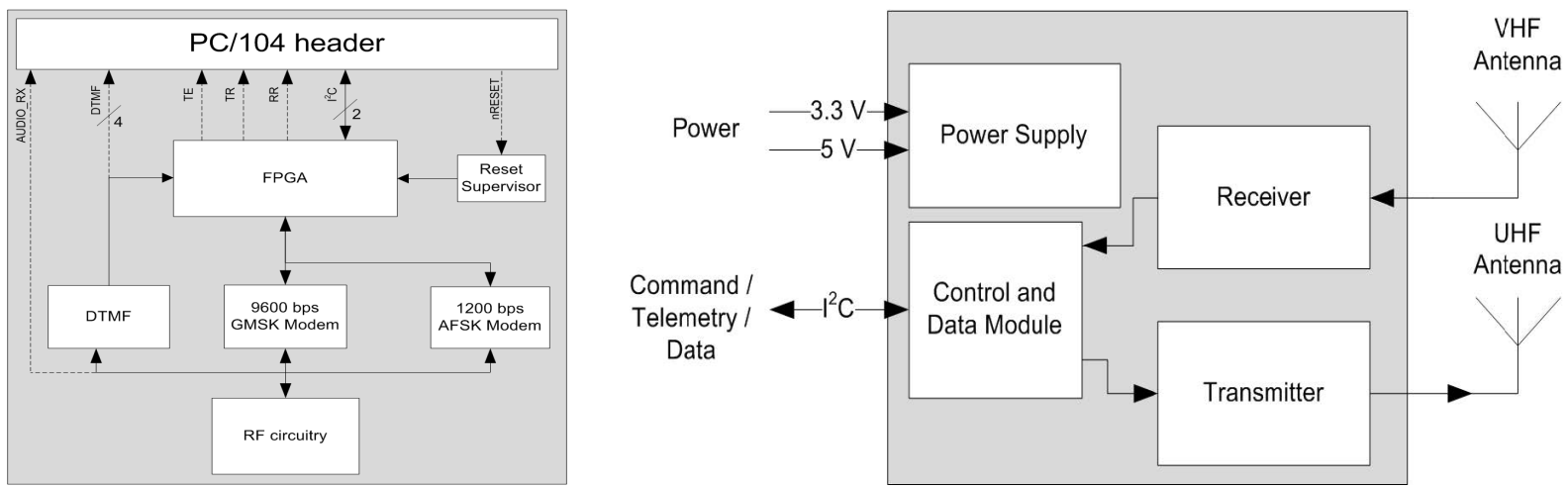

Fig. 1: Architecture of the VHF/UHF GMSK/AFSK modem

Table 1: Transmitter And ReCeiver features

\begin{tabular}{|l|l|l|l|l|l|l|l|}
\hline Mode & $\begin{array}{c}\text { Up/Down } \\
\text { link }\end{array}$ & $\begin{array}{c}\text { Modulation } \\
\text { (CRC-16-CCITT AX.25) }\end{array}$ & $\begin{array}{c}\text { Band } \\
\text { (Commercial) }\end{array}$ & $\begin{array}{c}\text { Frequency } \\
(\mathrm{MHz})\end{array}$ & $\begin{array}{c}\text { Channel spacing } \\
(\mathrm{kHz})\end{array}$ & $\begin{array}{c}\text { RF power } \\
(\mathrm{dBm})\end{array}$ & $\begin{array}{c}\text { Bit rate } \\
(\mathrm{bits} / \mathrm{s})\end{array}$ \\
\hline \hline 1 & $\mathrm{D}$ & GMSK & UHF & $400-420$ & 25 & $27-33$ & 9600 \\
\hline 2 & $\mathrm{D}$ & AFSK & UHF & $400-420$ & 25 & $27-33$ & 1200 \\
\hline 3 & $\mathrm{D}$ & GMSK & UHF & $400-420$ & 25 & $27-33$ & 9600 \\
\hline 1 & $\mathrm{U}$ & AFSK & VHF & $140-150$ & 12.5 & - & 1200 \\
\hline 2 & $\mathrm{U}$ & GMSK & VHF & $140-150$ & 12.5 & - & 9600 \\
\hline 3 & $\mathrm{U}$ & GMSK & $140-150$ & 12.5 & - & 9600 \\
\hline
\end{tabular}

mode exclusively. A variant of the transceiver operates in the amateur band.

\subsection{Signal Design for GMSK}

In GMSK modulation, the Non-Return-to-Zero (NRZ) binary pulse is shaped by a pre-modulation, Gaussian, lowpass filter which has an impulse response with no zero-crossings. The filtered baseband GMSK pulse model [7] is given as

$$
\begin{array}{r}
g(t)=\frac{1}{2 T}\left[\int_{t}^{\infty} \frac{1}{\sqrt{2 \pi}} e^{-\tau^{2} / 2} d \tau\right] \\
{\left[2 \pi B_{b}\left(\frac{t-T / 2}{\sqrt{\ln 2}}-\frac{t+T / 2}{\sqrt{\ln 2}}\right)\right]}
\end{array}
$$

where $T$ is the symbol period and $B$ is the $3 \mathrm{~dB}$ bandwidth. When the filter is not applied, bandwidthsymbol time product $B T \rightarrow \infty$ i.e., the modulation is MSK and the Gaussian shape of the pulse reduces to a rectangular shape: $g(t)=\frac{1}{2 T}$ for $0 \leq t \leq T$. The MSK power spectrum rises above the GMSK, loses content in the sidelobes and incurs penalty on the link quality through increase in the Occupied Channel BandWidth (OCBW) and Adjacent Channel Power Ratio (ACPR). The GMSK spectrum, however, is more compact and improves these metrics, but at the cost of higher InterSymbol Interference (ISI), SNR and per bit SNR $\left(E_{b} / N_{0}\right)$ at the receiver. Fig. 2 illustrates that the spectral density of GMSK attenuates sooner than MSK.

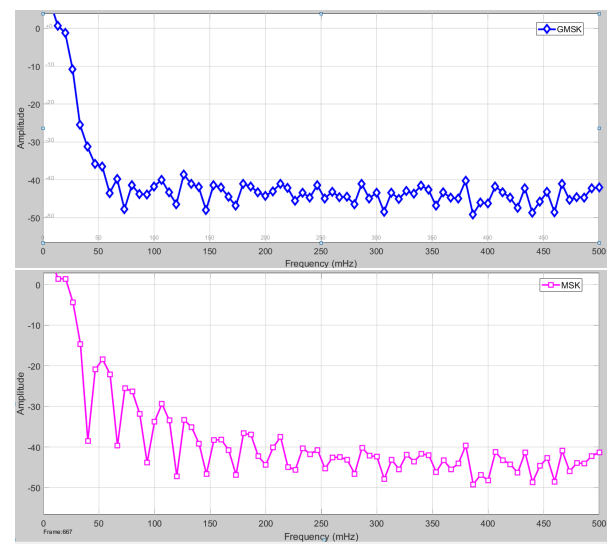

Fig. 2: GMSK $(B T=0.5)$ versus MSK

The impulse response of the Gaussian filter in GMSK is $f_{3 \mathrm{~dB}}=B T \cdot$ bit rate. For $B T=0.3$ the cutoff frequency ranges from $360 \mathrm{~Hz}$ to $2880 \mathrm{~Hz}$. When $B T$ is small, the pulse peaks and spreads over less number of bit periods and ISI increases. When $B T$ is large, the pulse attenuates but spreads over more number of bit periods, resulting in better ISI. For efficient transmissions, in a GMSK system, it is imperative to design the pulse for a nominal $B T$ to suppress the out-of-band spectrum leak- 
$0 \mathrm{~dB}$ gives $27 \mathrm{dBm}$ output power and $3 \mathrm{~dB}$ gain gives $33 \mathrm{dBm}$

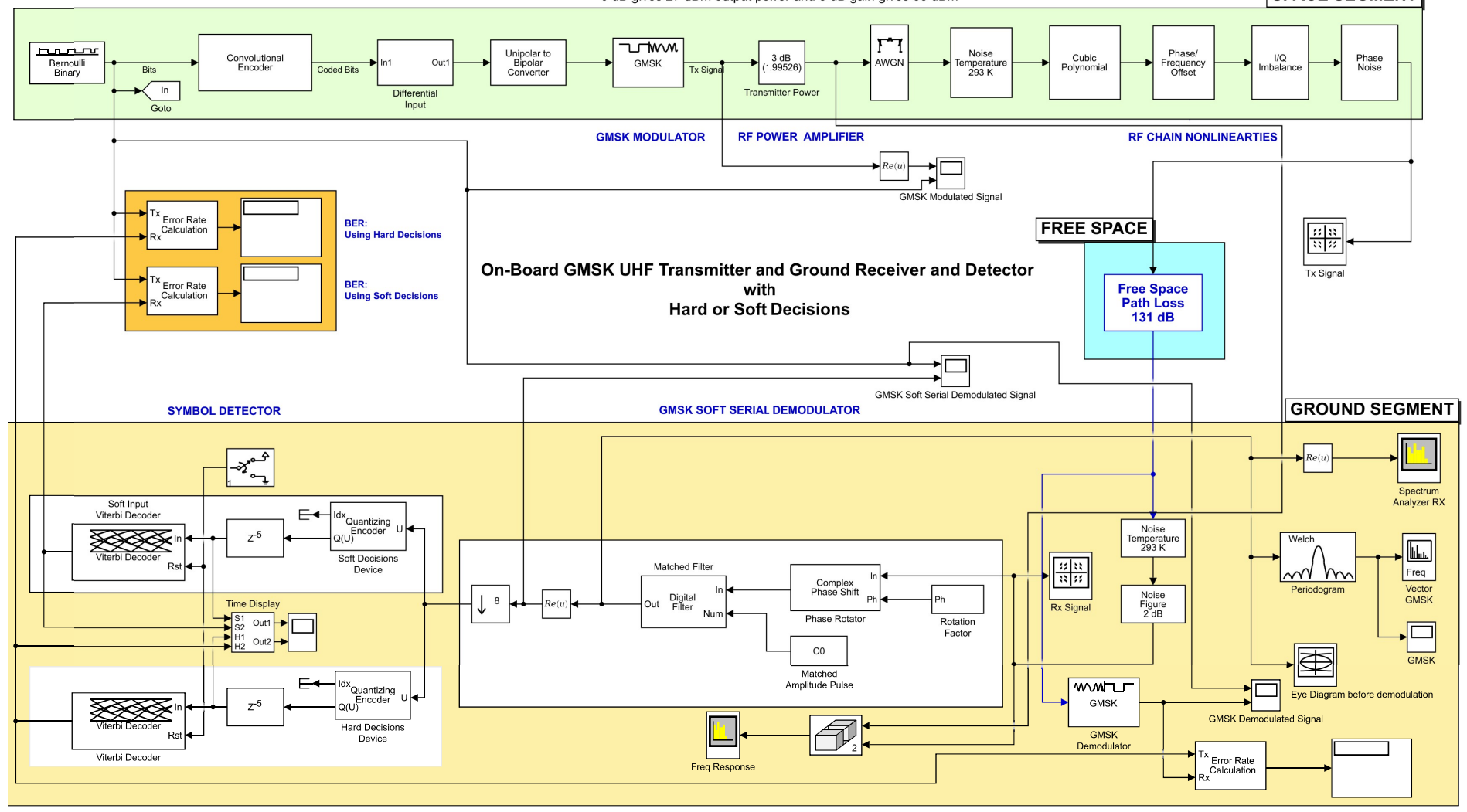

Fig. 3: System model of the on-board transmitter in space to ground link

age. For example, the European Telecommunications Standards Institute (ETSI) Global System for Mobile communications (GSM) specification imposes $B T=0.3$.

\subsection{Analysis Setup and the Simulation Model}

Fig. 3 shows the simulation model of the transmitter section. The RF power is adjustable in $27 \mathrm{dBm}, 30 \mathrm{dBm}$ and $33 \mathrm{dBm}$ levels.

In addition to the bandlimited nonlinear distortions present in the system, the channel is Additive White Gaussian Noise (AWGN), having zero mean noise with infinite bandwidth. To avoid receiver complexity in digital hardware in order to keep up with high, impractical sampling rates, the MSK demodulator is generally implemented as equivalent In-phase Quadrature (IQ) type orthogonal quadrature detector [8] in which the IQ signal variation is little compared to the phase variation of the carrier. Regardless of the occupancy of bandwidth, the average bit error probability (BEP) of coherent detectors whether BPSK, QPSK, OQPSK or precoded MSK type is equivalent [9]. This ideal situation occurs when the local oscillator phase and frequency are com- pletely synchronized to the received carrier. The BEP is given as

$P_{b}($ error $)=\sqrt{\frac{E_{b}}{N_{0}}}\left(\frac{1}{\sqrt{\pi}} \int_{t}^{\infty} e^{-\tau^{2}} d \tau\right)$

An estimate of the bit error rate in AWGN transmission for maximum likelihood quantized and unquantized detection and several modulator structures is shown in Fig. 4.

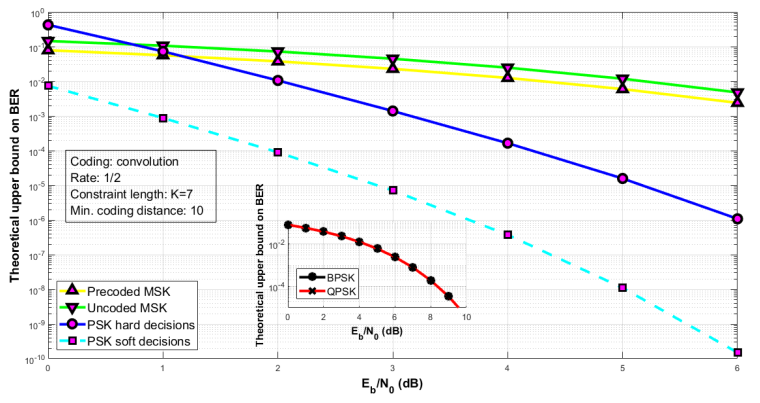

Fig. 4: Ideal bit error rate against the information bit energy to noise power spectral density ratio in AWGN environment 
The thermal noise power spectral density at a temperature $N_{0}$ is given as [10]

$N_{0}=k T \int_{0}^{\infty} \frac{h \omega / k T}{e^{h \omega / k T}-1} d \omega=-\frac{k^{2} T^{2}}{h} \int_{0}^{1} \frac{\ln x d x}{1-x}$

where $h$ is Planck's constant, $k$ is Boltzmann's constant and $T$ is the temperature, and $\omega$ is the frequency band under consideration. Note that $\frac{h \omega}{k T} \ll 1$. The thermal noise power varies according to Eq. 4 in the thermal cycle from $3.02 \times 10^{-8} \mathrm{~W}$ to $4.93 \times 10^{-8} \mathrm{~W}$.

$$
P_{n}(T)=\frac{\pi^{2}}{6} \frac{(k T)^{2}}{h}
$$

The Free Space Path Loss (FSPL) is taken at $600 \mathrm{~km}$ altitude.

$$
F S P L=\left[\frac{4 \pi d f}{C}\right]^{2}
$$

where $d$ the distance between transmitter and receiver and $f$ the signal frequency are in $\mathrm{Hz}$ and $\mathrm{m}$ respectively, and $C$ is speed of light in vacuum.

The periodogram of the output signal is shown in Fig. 5. The 2 nd harmonic occurs at $-40 \mathrm{dBc}$ above which and up to the fundamental is the clear signal space known as the Spurious Free Dynamic Range (SFDR). The model has been setup in a way that the largest spur happens to be the 2nd harmonic. This has been the case according to the empirical data as we shall see in Section 3.

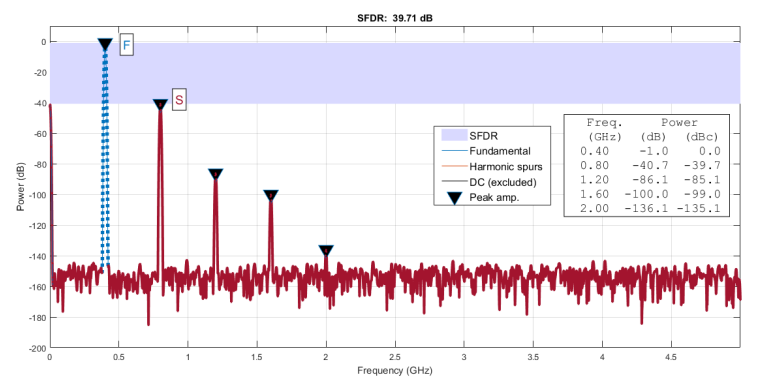

Fig. 5: SFDR is the signal range available as the difference in amplitudes of the fundamental and the largest spur that is clear of any nonlinearities

Various nonlinearities contributed by the Power Amplifier (PA) are modeled which distort the signal and add offsets in the carrier frequency, phase and the IQ gain. The PA is modeled using a truncated power series polynomial $P$ having peak voltage amplitude $A$. The model suits a weak nonlinear device giving off only static, memoryless, nonlinearity to an instantaneous input. This is so as the PA on the transceiver has been organized to operate in the linear range of amplitude-amplitude modulation (AM-AM conversion).

The polynomial shown in Eq. 6 describes the spectral contributions in the amplifier output.

$$
\begin{array}{r}
P=\underbrace{a_{2} / 2 A^{2}}_{\text {DC bias }}+\underbrace{a_{1} A}_{\text {linear gain }}+\underbrace{a_{2} / 2 A^{2}}_{\text {2nd harmonic }}+\underbrace{a_{3} A^{3}}_{\text {3rd order distortion }} \\
P=\underbrace{a_{2} / 2 A^{2}}_{\text {DC bias }}+\underbrace{a_{1} A+3 a_{3} / 4 A^{3}}_{\text {fundamental }}+ \\
\underbrace{a_{2} / 2 A^{2}}_{\text {2nd harmonic }}+\underbrace{a_{3} / 4 A^{3}}_{\text {3rd harmonic }}
\end{array}
$$

where coefficients $a_{k}$ of the Taylor series expansion are real constants. On a real amplifier, these coefficients can be determined in laboratory on AM-AM power sweep test by employing curve fitting techniques on the measured trace on a signal analyzer.

Considering the input signal to be narrowband and assuming the amplifier transfer characteristics are frequency independent [11], the amplifier output is digitally corrected by gauging the input as a linear ramp. To start the approximation using the distortion data, a 3rd degree polynomial is numerically fitted, with the least square method, to an input of linear ramp. The amplifier output $P(x)$ comprising of harmonic powers in amplitude $x$, and the coefficients given in Eq. 7 are computed. Note the dominance of linear gain, followed by the bias and the 2nd harmonic, and small dynamics of the nonlinearity.

$P(x)=0.0007 x^{3}+0.0164 x^{2}+0.9994 x-0.0200$

The corrected response is in Fig. 6 showing that the harmonics now have been significantly rarified.

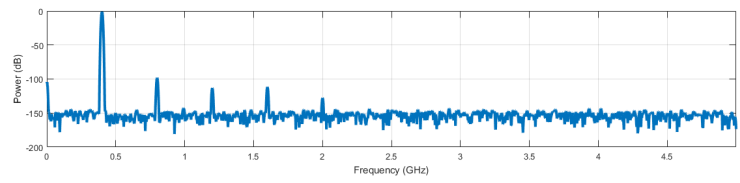

Fig. 6: Polynomial corrected harmonics

The resulting dynamic range through the correction and improved spectral purity is then determined by estimating the SINAD, SNR and Total Harmonic Distortion (THD) metrics against a sweep of input attenuation. The optimum metrics thus obtained are plotted in Fig. 7. 


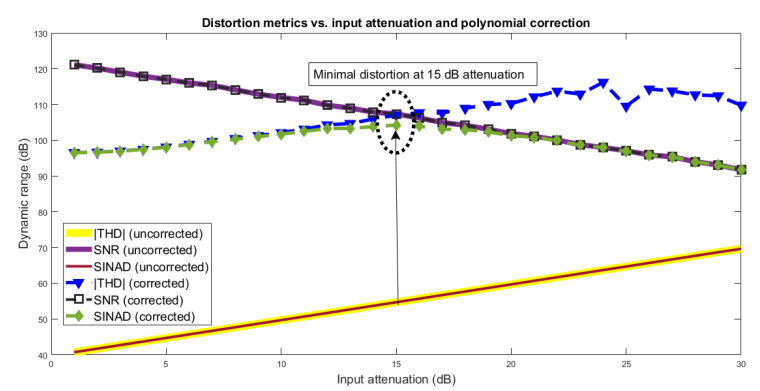

Fig. 7: Dynamic range against the input attenuation when polynomial correction is applied

The use of attenuator models has bearing on the load being driven and tradeoff between the load and the tolerable attenuation level becomes necessary. As shown in Fig. 7, it is expected that the power loss to an ideal load would occur around $15 \mathrm{~dB}$ at which the dynamic figures of merit tend to be the most favorable.

The strongest TX signal and expected upper ACPR is shown in Fig. 8. The ACPR is about $38 \mathrm{dBc}$ down at full power. Therefore, the strength of spectral growth may be expected to be insignificant, chiefly due to the linear amplification.

At the ground station, the received signal is modeled for sensitivity level of $-129 \mathrm{dBm}$. Fig. 9 shows the sensitivity.

\subsection{Thermal Analysis}

Thermal Finite Element Analysis (FEA) of the transceiver board shows the temperature field depicted in Fig. 10 and at least $70^{\circ} \mathrm{C}$ below the PA die surface of the board. In addition to the thermal plane in the PCB stack-up, the thermal gradient is readily counterbalanced using an enclosure which will also act as the $\mathrm{RF}$ shield for protection for the radiated immunity of the RF front-end.

An Infra-Red (IR) temperature gun is used to infer the temperature of the hot spots on the actual board at UHF frequency under nominal RF test conditions. Fig. 11 shows this imagery.

\section{Transmitter Characterization}

The performance of radio is evaluated with a test setup that is shown in Fig. 12. The radio transmission is analyzed over the thermal cycle using HP8920 RF communications testset. The FM reference applied is ANSI / TIA / EIA-RS-316-B, RS-152-C. The test frequency range is $25 \mathrm{MHz}$ to $1000 \mathrm{MHz}$ and $25 \mathrm{MHz}$ to $866 \mathrm{MHz}$.
An automation software [12] loads the radio configuration using an Arduino device over the $\mathrm{I}^{2} \mathrm{C}$ interface to the radio FPGA and also performs the electrical and functional tests in the thermal cycle. The configuration includes: up and downlink modulation schemes, TX and RX frequencies, and up and down data rates. The thermal chamber by Binder is communicated over the Modbus tunnel on top of LAN. The electrical test equipment communicates over the GPIB bus to the automation software developed in LabWindows/CVI. Channel currents are measured first.

\subsection{DC and RF Power Characterization}

The health monitoring of the transceiver board is illustrated in Fig. 13. The board telemetry is updated once every second. The Switched Mode Power Supply (SMPS) and PA temperature sensors are located close to the respective components they monitor, namely the SMPS and PA ICs. The temperature is monitored during transmission. The SMPS and PA temperature registers contain the temperature readings of the most recent conversions. Negative values are returned in two's complement. The conversion range is from $-25^{\circ} \mathrm{C}$ to $+127^{\circ} \mathrm{C}$.

Table 2 lists the power consumption when measured in the thermal cycle. On the average, at $0.5 \mathrm{~W}, 1 \mathrm{~W}$ and $2 \mathrm{~W}$ transmit levels and the receiver also running, the drift in power consumption is $3 \%, 0.8 \%$ and $1.6 \%$ respectively, while the receiver consumption swings as much as $10 \%$ in the cycle. Both the receiver and transmitter tend to consume less power in the cooler end of the thermal cycle.

The output at different transmit levels is listed in Table 3. Overall, the maximum variation in the $\mathrm{RF}$ power is no more than $1.1 \mathrm{dBm}$ in the thermal cycle. From both tables, it is clear that the nominal performance of the transceiver may be expected in $1 \mathrm{~W}$ mode.

Within the thermal cycle, the relationship of the DC and RF power at different transmitting levels can be seen in Figs. 14 and 15.

\subsection{FM Deviation}

The FM stability of the output modulation is measured over temperature as the maximum difference between the modulated frequency and the carrier central frequency. Table 4 shows the average test results in thermal cycle. The strong stability is likely to compact the OCBW and spectral leakage according to the Carson's rule [13] as 


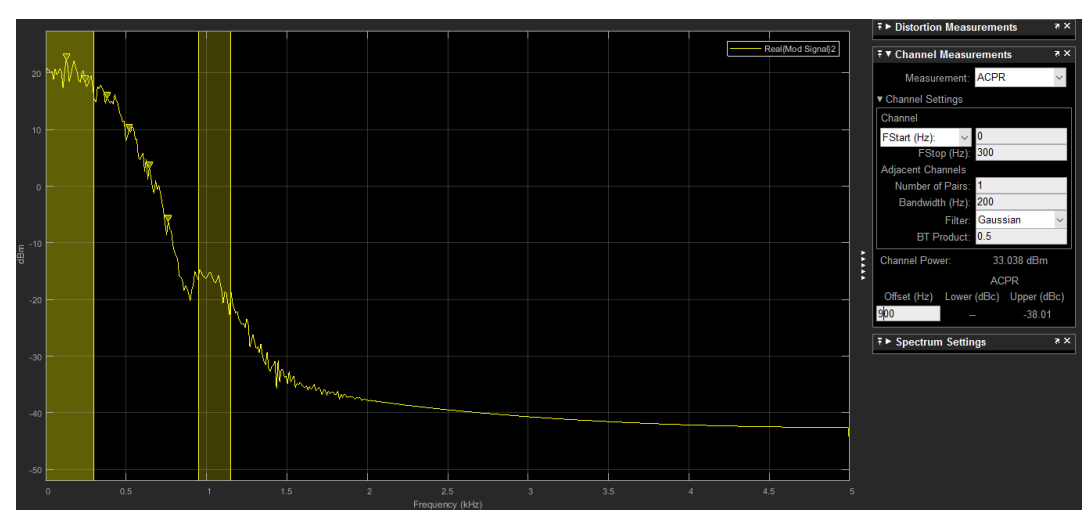

Fig. 8: On-board transmitted signal at $33 \mathrm{dBm}$ and the power leakage

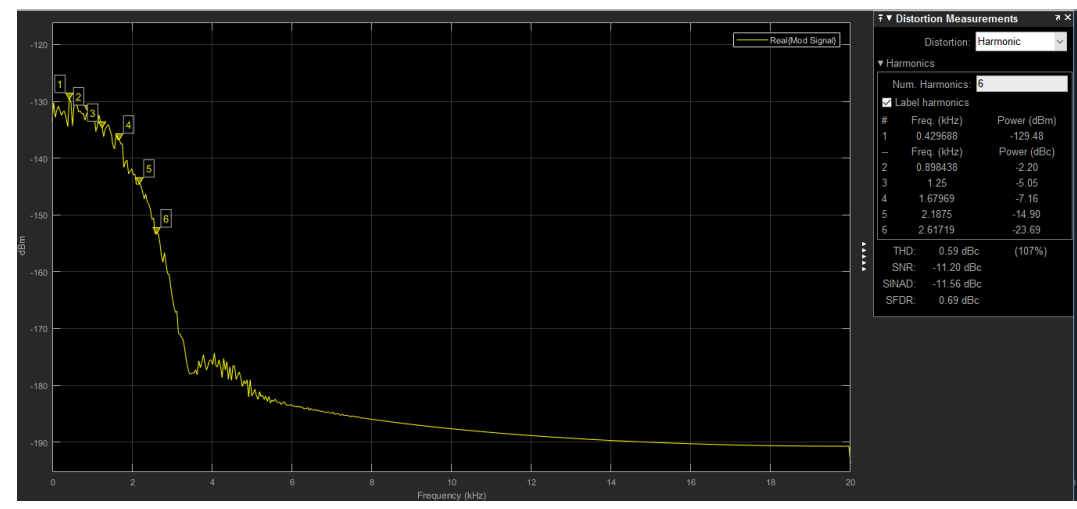

Fig. 9: Ground received signal spectrum

Table 2: Transmitter CURREnT DRAW AT DIFFEREnT TEMPERATURES

\begin{tabular}{|l|l|l|l|l|l|l|}
\hline \multicolumn{6}{|c|}{ Typical power consumption in the transmitter } \\
\hline \hline Temperature & -21 & -5 & 15 & 35 & 51 & ${ }^{\circ} \mathrm{C}$ \\
\hline \hline Current (5 V) & 555 & 558 & 563 & 565 & 569 & $\mathrm{~mA}$ \\
\hline $0.5 \mathrm{~W}$ & 2976 & 2995 & 3026 & 3043 & 3069 & $\mathrm{~mW}$ \\
\hline Current (5 V) & 807 & 809 & 812 & 810 & 803 & $\mathrm{~mA}$ \\
\hline $1 \mathrm{~W}$ & 4236 & 4250 & 4271 & 4268 & 4239 & $\mathrm{~mW}$ \\
\hline Current (5 V) & 1187 & 1202 & 1205 & 1190 & 1192 & $\mathrm{~mA}$ \\
\hline $2 \mathrm{~W}$ & 6136 & 6215 & 6236 & 6168 & 6184 & $\mathrm{~mW}$ \\
\hline
\end{tabular}

Table 3: TRANSMITTER RADIO POWER MEASUREMENTS AT DIFFERENT TEMPERATURES

\begin{tabular}{|c|c|c|c|c|c|c|c|}
\hline \multicolumn{8}{|c|}{ Typical output RF power } \\
\hline Frequency & \multicolumn{2}{|c|}{$\overline{401}$} & \multicolumn{2}{|c|}{410} & \multicolumn{2}{|c|}{ 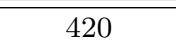 } & $\mathrm{MHz}$ \\
\hline \multicolumn{8}{|c|}{$-5^{\circ} \mathrm{C}$ to $+35^{\circ} \mathrm{C}$} \\
\hline Output RF & $\min$ & $\max$ & $\min$ & $\max$ & $\min$ & $\max$ & $\mathrm{dBm}$ \\
\hline $0.5 \mathrm{~W}$ & 27.1 & 27.2 & - & - & - & - & $\mathrm{dBm}$ \\
\hline $1 \mathrm{~W}$ & 29.97 & 30.1 & - & - & - & - & $\mathrm{dBm}$ \\
\hline $2 \mathrm{~W}$ & 32.35 & 32.5 & 32.1 & 32.3 & 31.5 & 31.9 & $\mathrm{dBm}$ \\
\hline \multicolumn{8}{|c|}{$-21^{\circ} \mathrm{C}$ to $+51^{\circ} \mathrm{C}$} \\
\hline Output RF & $\min$ & $\max$ & $\min$ & $\max$ & $\min$ & $\max$ & $\mathrm{dBm}$ \\
\hline $0.5 \mathrm{~W}$ & 27.1 & 27.3 & - & - & - & - & $\mathrm{dBm}$ \\
\hline $1 \mathrm{~W}$ & 29.9 & 30.1 & - & - & - & - & $\mathrm{dBm}$ \\
\hline $2 \mathrm{~W}$ & 32.4 & 32.6 & 32.1 & 32.4 & 31.5 & 32.0 & $\mathrm{dBm}$ \\
\hline
\end{tabular}



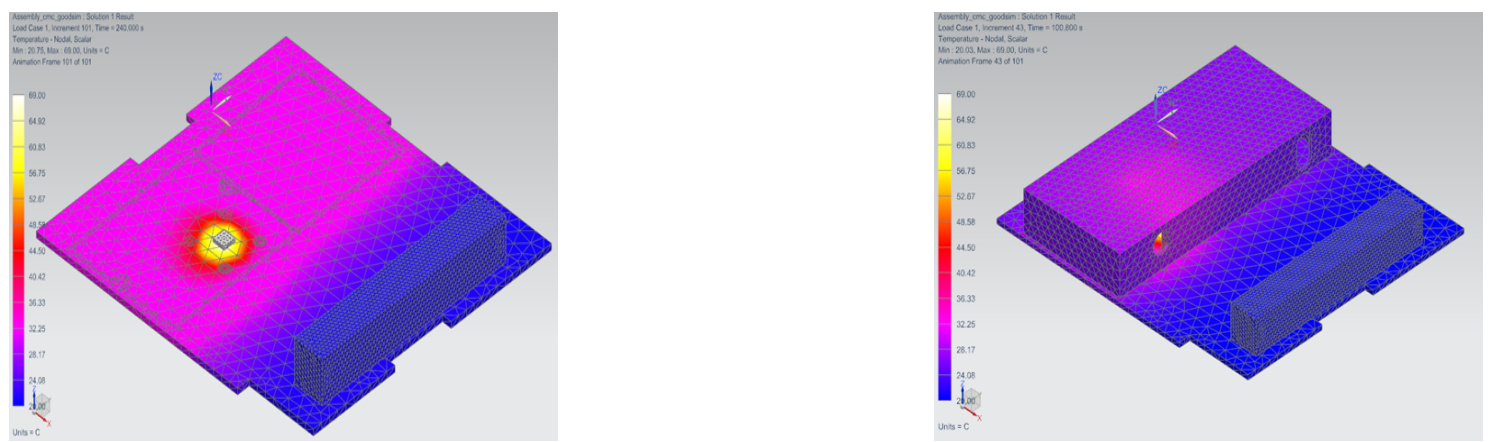

Fig. 10: Predicted temperature gradient of the transceiver around the PA area, without enclosure and with enclosure
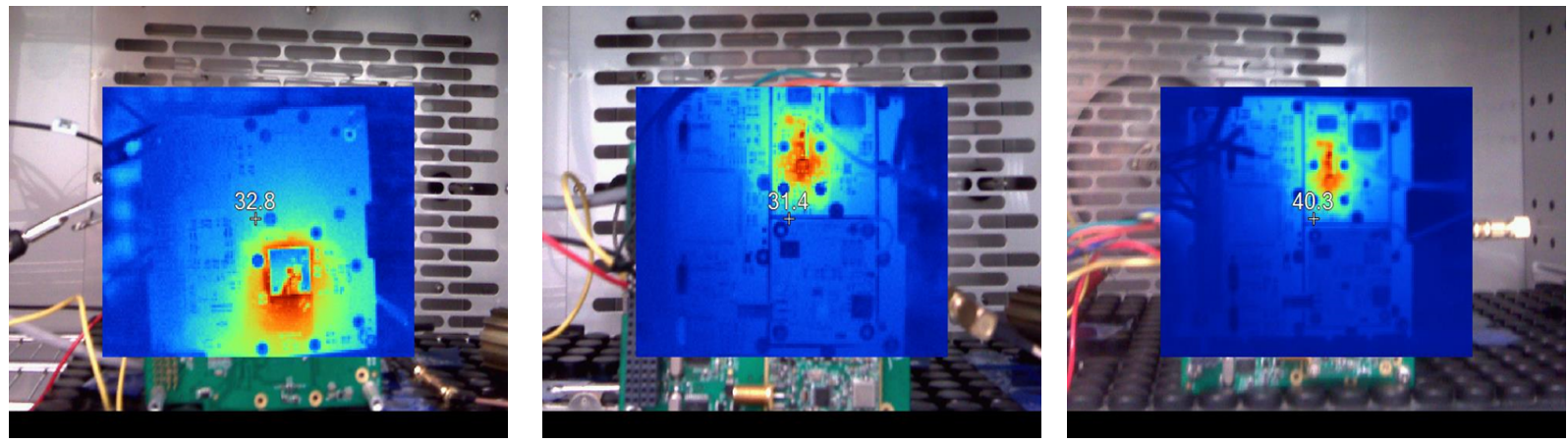

Fig. 11: Thermal scan of the transceiver card. Top (left), bottom (center) views and an inductor as heating source (right)

$O C B W=2\left(f_{\text {dev }}+f_{\text {mod }}\right)$

Table 4: CARrier Stability OVER temperature RANGeS

\begin{tabular}{|l|l|l|}
\hline \multicolumn{2}{|c|}{ Deviation in the modulated frequency (410 MHz) } \\
\hline \hline Max specified deviation & \multicolumn{2}{|c|}{$3 \mathrm{kHz}$} \\
\hline Temperature range & Drift (ppm) & Frequency $(\mathrm{Hz})$ \\
\hline$-5{ }^{\circ} \mathrm{C}$ to $+35^{\circ} \mathrm{C}$ & 2.7 & 1107 \\
$-21^{\circ} \mathrm{C}$ to $+51{ }^{\circ} \mathrm{C}$ & 3.2 & 1312 \\
\hline
\end{tabular}

\subsection{Spectral Purity and Spurs}

The spectrum is searched for the presence of harmonics, harmonic products, nonharmonics or any other spurious component under a single tone test. The center frequency is tuned in $1 \mathrm{MHz}$ steps from $400 \mathrm{kHz}$ to $1000 \mathrm{MHz}$ on the testset. Furthermore, to isolate susceptibility to any interferer in the vicinity, the source of the emissions is verified to be the transceiver itself. This is done by unkeying the transmitter.

Table 5 lists the levels of the dominant harmonics found in the spectrum at the maximum transmit level and within the thermal cycle. These levels are fairly consistent and low enough throughout the cycle. Also, they confirm the amplifier being weakly nonlinear. The 2nd order spurious response, generally, slightly worsens at the hot end of the thermal cycle. This observation is true for all power levels as indicated in Fig. 16.

Table 5: MeAsured harmonic LeVELS

\begin{tabular}{|c|c|c|c|}
\hline \multicolumn{4}{|c|}{ Typical 2nd harmonic levels } \\
\hline \hline Power & $\min$ & $\max$ & below carrier \\
\hline \multicolumn{4}{|c|}{$-5^{\circ} \mathrm{C}$ to $+35^{\circ} \mathrm{C}$} \\
\hline $2 \mathrm{~W}$ & -40.7 & -39.0 & $\mathrm{dBc}$ \\
\hline \multicolumn{4}{|c}{$-21^{\circ} \mathrm{C}$ to $+51^{\circ} \mathrm{C}$} \\
\hline \hline $2 \mathrm{~W}$ & -41.6 & -39.0 & $\mathrm{dBc}$ \\
\hline
\end{tabular}

At different power modes, the effects of variation in $\mathrm{RF}$ power, carrier frequency and the spurious response in the thermal cycle are plotted in unison in Fig. 17. The level of $2 \mathrm{nd}$ harmonic gives insight to the DC component in the carrier as their amplitudes are equal, caused by the square law term $\left(a_{2} A^{2} / 2\right)$ in the power series (Eq. 6). The DC component is at least $-7.7 \mathrm{dBm}$ or $92 \mathrm{mV}$. 


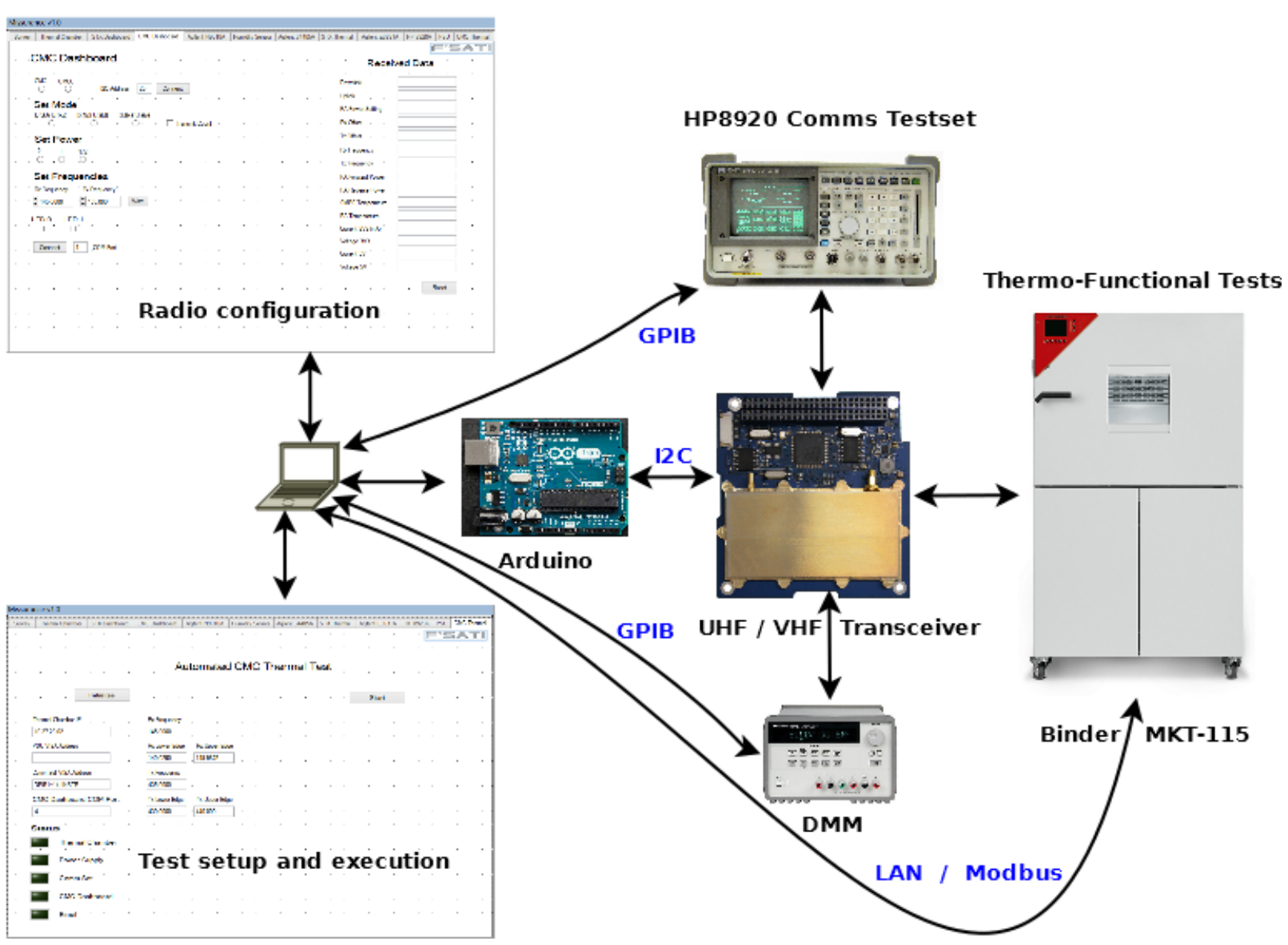

Fig. 12: Test and measurement testbed

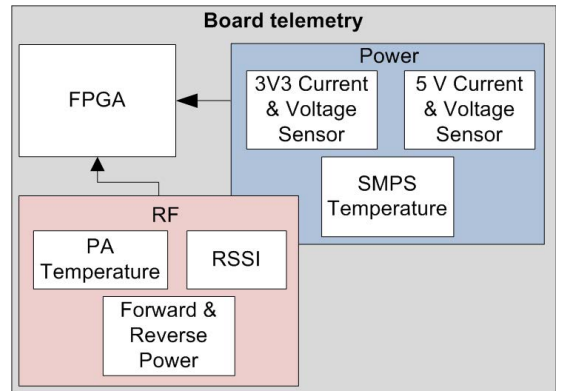

Fig. 13: Transceiver on-board telemetry collection

\subsection{Forward and Reverse Power}

The forward and reverse power registers can be read via the $\mathrm{I}^{2} \mathrm{C}$ telemetry interface (Fig. 13). These registers are used to calculate the forward and reverse $\mathrm{RF}$ transmit power, as well as the return loss, and thus, indicate any mismatch in impedance between the transmitter and the antenna. That is, the transmitter may be calibrated for possible antenna Voltage Standing Wave Ratio (VSWR) conditions. The registers contain 12-bit values of the

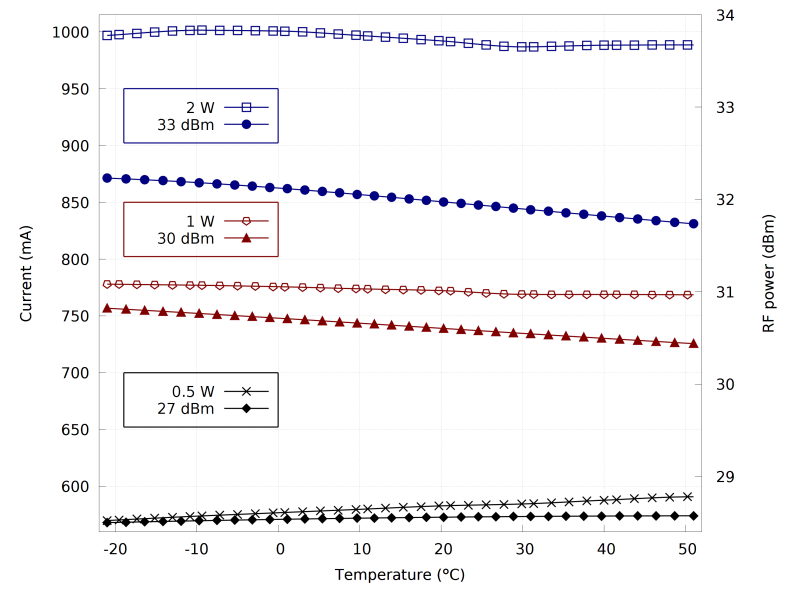

Fig. 14: Input DC and output RF power of the transmitter at different modes in the thermal cycle

PA's forward and reverse power which are converted into voltages as

$$
V_{\text {rvs|fwd }}=(\text { value })_{d} \times \frac{3}{4096}
$$

The coupled forward or reverse power is obtained as 

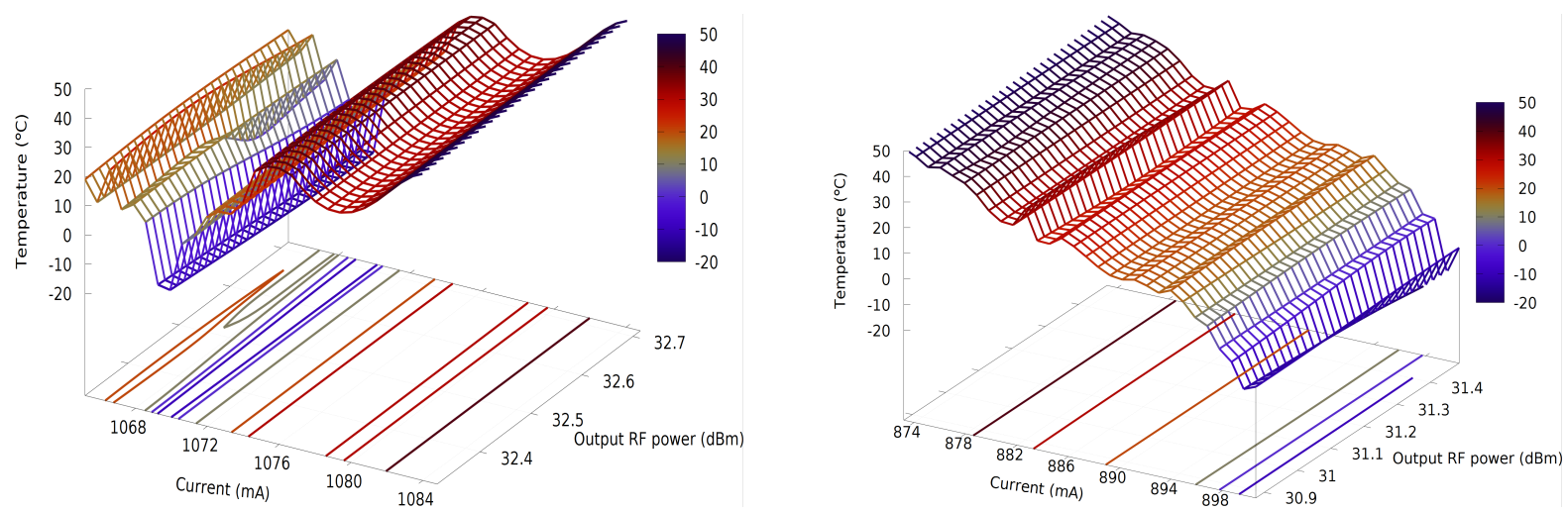

Fig. 15: Transmitter current at $410 \mathrm{MHz}$ (left) and $420 \mathrm{MHz}$ (right) when RF output is at $33 \mathrm{dBm}$ in the thermal cycle
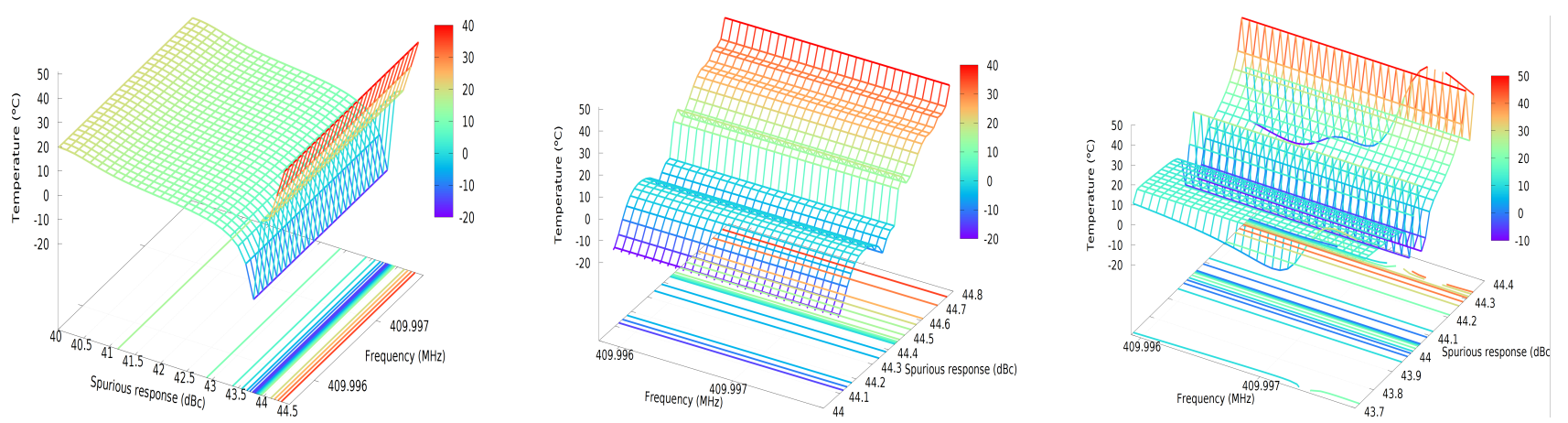

Fig. 16: 2nd harmonic spur and variation in transmitter frequency of $410 \mathrm{MHz}$ at $27 \mathrm{dBm}$ (left), $30 \mathrm{dBm}$ (middle) and $33 \mathrm{dBm}$ (right) power levels in the thermal cycle

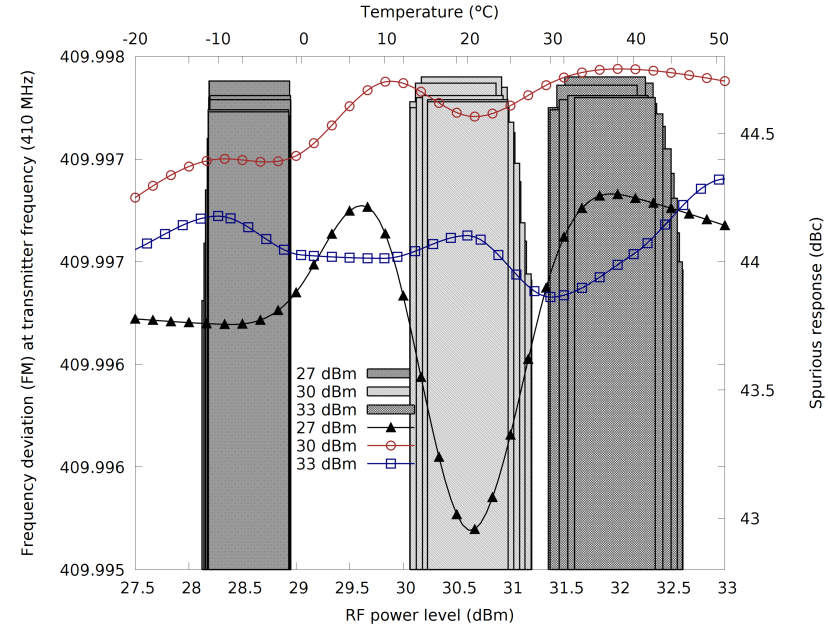

Fig. 17: At the three output power levels the deviation in transmitter frequency is $<3 \mathrm{kHz}$ throughout the thermal cycle when the channel is centered at $410 \mathrm{MHz}$

$$
\begin{gathered}
P_{\text {coupled fwd } \mid r v s}=-68838 V_{\text {fwd|rvs }}{ }^{6}+ \\
228000 V_{f w d \mid r v s}{ }^{5}-308831 V_{f w d \mid r v s}{ }^{4}+ \\
218934 V_{f w d \mid r v s}{ }^{3}-85741 V_{f w d \mid r v s}{ }^{2}+ \\
17660 V_{\text {fwd|rvs }}-1511.8 \quad \text { (dB) }
\end{gathered}
$$

The actual forward or reverse power is obtained by adding $32.5 \mathrm{dBm}$ to $P_{\text {coupled fwd|rvs }}$. The spread of forward and reverse power is illustrated through the ovals in Fig. 18 at the respective transmitting levels.

\subsection{Return Loss}

The return loss is the difference of the actual forward and the actual reverse power, i.e.,

Return Loss $=-10 \log _{10} \frac{P_{\text {reflected }}}{P_{\text {fwd }}}$

The losses measured at several attenuation levels of the signal using directional couplers are displayed in Fig. 19. The return losses are $13.86 \mathrm{~dB}, 14.18 \mathrm{~dB}$ and $14.10 \mathrm{~dB}$ at the $27 \mathrm{dBm}, 30 \mathrm{dBm}$ and $33 \mathrm{dBm}$ respectively. Recall the simulation on selecting the best attenuator had shown a $15 \mathrm{~dB}$ attenuator (Fig. 7). The discrepancy may be improved by carefully choosing better coefficients of the polynomial. 


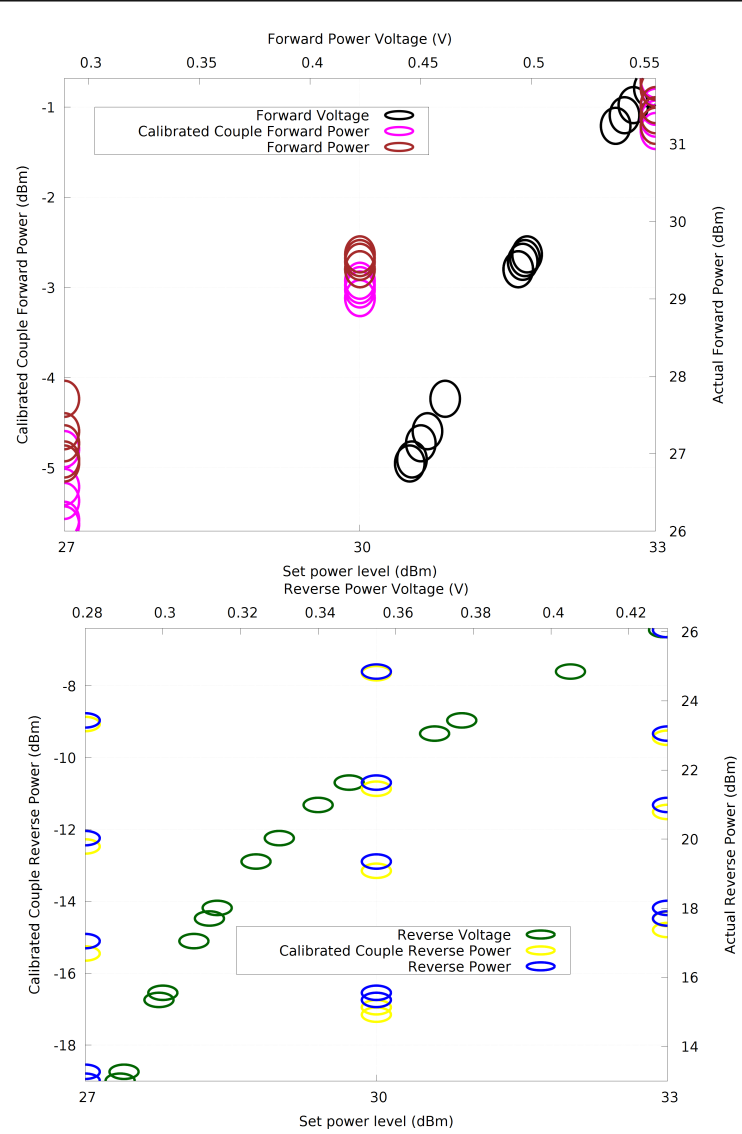

Fig. 18: Calibration using couplers for forward and reverse power

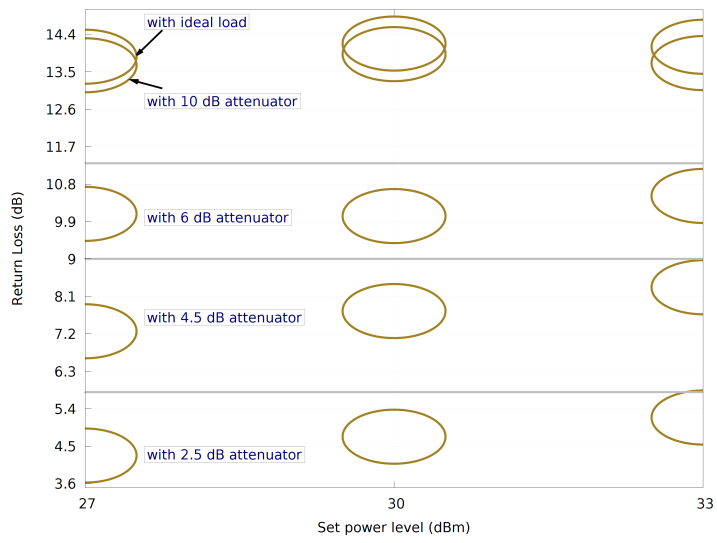

Fig. 19: Return loss with directional couplers in place to calibrate forward and reverse power

\subsection{Amplifier Nonlinearity}

Although GMSK is insensitive to phase distortion and nonlinearities, it is still prudent to operate the PA in small-signal (linear) region for radiated emissions control, most notably, for better ACP and reducing the spectral spread measured as the OCBW. There is a large, linear, dynamic operating range available in the PA which is utilized for the aforementioned purposes.

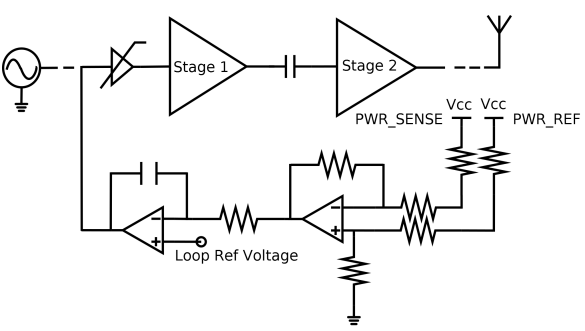

Fig. 20: InGaP heterojunction bipolar transistor technology PA, stages and feedback linear gain adjustment

A basic block diagram of the amplifier circuit is in Fig. 20. PWR_REF returns a voltage proportional to the DC component of the drive current in the output stage and PWR_SENSE contributes to the RF components as well the DC. The difference of the current components yields the RF only current and the resultant RF power. The logarithmic difference and the RF power maintains a linear relationship up to $33.5 \mathrm{dBm}$. This is the upper limit of linearity to which we operate at maximum $2 \mathrm{~W}$ mode. Beyond $34.5 \mathrm{dBm}$, which is the beginning of the $1 \mathrm{~dB}$ compression point and with the employed power control scheme, the amplifier may saturate, gain flattening may be seen or even constriction may appear, unless a different control scheme e.g., hybrid couplers and input/output matching networks are used in a balance amplifier configuration.

\subsection{Amplifier Efficiency}

The price of linearity is paid in efficiency. Fig. 21 shows the amplifier efficiencies tabulated at different transmit levels. A maximum efficiency of $33.5 \%$ is observed at the coldest end of the thermal cycle in $2 \mathrm{~W}$ mode. Even at the hottest temperature and in the least power mode, the amplifier is at least $24.5 \%$ efficient.

\section{Receiver Characterization}

The receiver current varies $11 \%$ during the thermal cycle. The measurements are shown in Table 6 which improve at low thermal noise in the cold end of the cycle. 


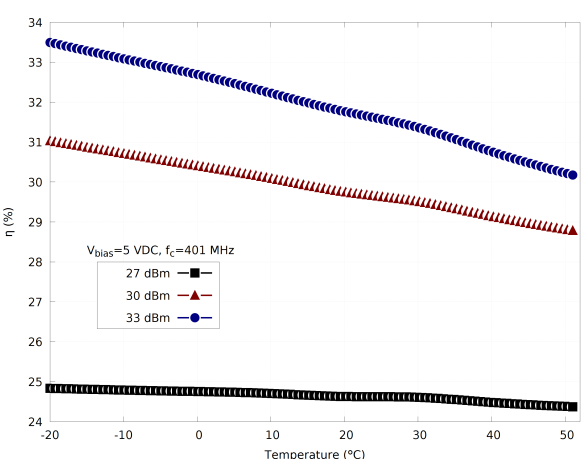

Fig. 21: Amplifier efficiency lowers $10 \%, 7 \%$ and $1.6 \%$ between the cold and hot extremes of the thermal cycle at respective $33 \mathrm{dBm}, 30 \mathrm{dBm}$ and $27 \mathrm{dBm}$

Table 6: ReCEIVER CURRENT DRAW AT DIFFERENT TEMPERATURES

\begin{tabular}{|l|l|l|l|l|l|l|}
\hline \multicolumn{7}{|c|}{ Typical power consumption in the receiver } \\
\hline \hline Temperature & -21 & -5 & 15 & 35 & 51 & ${ }^{\circ} \mathrm{C}$ \\
\hline \multicolumn{7}{|c|}{ Idle state } \\
\hline \hline Current (3.3 V) & 61 & 62 & 64 & 66 & 68 & $\mathrm{~mA}$ \\
\hline Current (5 V) & \multicolumn{7}{|c|}{$<100$} \\
\hline \multicolumn{7}{|c|}{ Receiver ON } \\
\hline \hline Receive only & 201 & 205 & 211 & 218 & 224 & $\mathrm{~mW}$ \\
\hline
\end{tabular}

4.1 Minimum Discernible Signal (MDS) and Spurious Free Dynamic Range (SFDR)

The receiver front-end Noise Figure (NF) is measured to be less than $1.5 \mathrm{~dB}$. This yields an equivalent noise floor in a $12.5 \mathrm{kHz}$ channel according to

$$
\text { Noise Floor }=N F+10 \log _{10}[\Delta f k T]
$$

The noise floor sits at $-132 \mathrm{dBm}$ to $-131 \mathrm{dBm}$ at the extremes from $-20^{\circ} \mathrm{C}$ to $+50^{\circ} \mathrm{C}$. The noise floor is the minimum discernible signal level.

Upon evaluation of several boards, the SFDR is specified at $-120 \mathrm{dBm}$. The extreme nonlinearity of the receiver is then estimated as the Input Intercept Point of 3rd order $\left(\mathrm{IIP}_{3}\right)$, shown in Eq. 13, which includes not only the harmonics and their variants but also the Intermodulation (IM) product differences of any interferer with the carrier.

$I I P_{3}=\frac{3}{2} S F D R+$ Noise Floor $\left.\right|_{+50{ }^{\circ} \mathrm{C}}$

The $\mathrm{IIP}_{3}$ turns up around $-49 \mathrm{dBm}$. This figure is quite low, for example, compared to GSM-900 service. The reason is the absence of interferes such as the co-located subscribers inducing IM distortion in the terrestrial mobile radio services.

\subsection{RSSI}

The Receive Signal Strength Indicator (RSSI) telemetry (Fig. 13) is a 12-bit value returned over two bytes. The RSSI provides an indication of the current received signal strength. This telemetry should typically be captured when a signal is being received from the ground. Table 7 provides an indication of the RF level given as the output voltage. The higher the received voltage the better the RF signal being received. Note that this is a noisy telemetry channel unless a carrier signal is present.

Table 7: RSSI VOLTAGE LEVELS

\begin{tabular}{|l|l|l|l|}
\hline \multicolumn{4}{|c|}{ RSSI levels } \\
\hline \hline RF power $(\mathrm{dBm})$ & $\min (\mathrm{V})$ & typical $(\mathrm{V})$ & $\max (\mathrm{V})$ \\
\hline-118 & - & 0.3 & 0.8 \\
\hline-68 & 0.7 & 1.1 & 1.8 \\
\hline-23 & 1.2 & 1.8 & 2.5 \\
\hline
\end{tabular}

The conversion is according to Eq. 14.

$$
\text { RSSI Voltage }=(\text { value })_{d} \times \frac{3}{4096}
$$

\section{$4.312 \mathrm{~dB}$ SINAD}

The FM receiver's SIgnal Noise And Distortion (SINAD) ratio at the output of the receiver is defined as

$$
S I N A D=10 \log _{10}\left[\frac{\text { signal }[V]^{2}+\text { noise }[V]^{2}+\operatorname{distort}[V]^{2}}{\text { noise }[V]^{2}+\text { distort }[V]^{2}}\right]
$$

Effectively, the SINAD is SNR without the harmonics. Typical specifications, rooted in the law enforcement agencies and emergency vehicle audio communications intelligibility, call for $12 \mathrm{~dB}$ [14]. The level corresponds to $4: 1$ at which the contribution of noise and distortion is $25 \%$ to the signal amplitude.

The receiver input is fed with a VHF frequency signal that is modulated with a $1 \mathrm{kHz}$ tone that introduces spurs and distortions in the output. At the output, the SINAD meter of HP8920 measures the power of the modulating tone and the noise and distortion. The power of the modulating tone is filtered out and the noise and distortion power is measured in the channel bin. The ratio of the two power measurements is the SINAD. A meter averaging function is used for 10 readings to measure a consistent signal quality. 


\subsection{2 dB SINAD Sensitivity}

The minimum level of the modulated radio carrier input that is required to yield $12 \mathrm{~dB}$ SINAD ratio at the receiver at specific power output is the receiver sensitivity. During the SINAD test, the amplitude of the receiver input is adjusted such that the SINAD reads $12 \mathrm{~dB}$. This level of the receiver input signal is the $12 \mathrm{~dB}$ SINAD sensitivity.

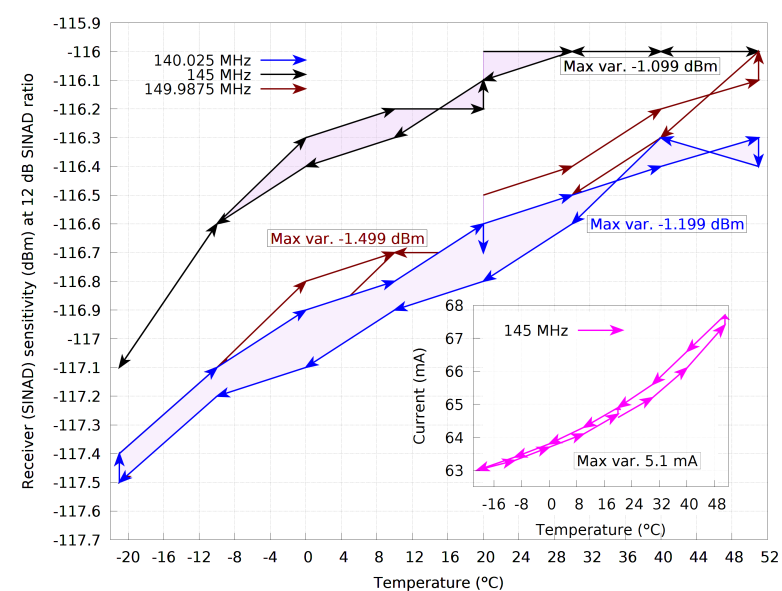

Fig. 22: Variation in receiver sensitivity at upper, lower and center frequencies in the thermal cycle

The sensitivity is measured during the full thermal cycle at three VHF frequencies. On the whole, the level turns out to be $-117 \mathrm{dBm}$ and is rather insensitive to the variation in temperature. This is illustrated in Fig. 22. The largest variation is found at the highest frequency of the channel. The test reference applied is EIA RS-204-D.

Although not exclusively measured, an estimate of the noise and distortion content at the sensitivity level, and its variation in the thermal cycle may be established through Fig. 23. The typical contribution of noise and distortion is around $-146 \mathrm{dBm}$. That is, about $30 \mathrm{dBc}$ below the sensitivity.

\subsection{Receiver $E b / N_{0}$}

Using the $12 \mathrm{~dB}$ SINAD (instead of SNR), we estimate worst case $E b / N_{0}$ at the receiver sensitivity and when the bit rate is maximum. It turns out to be $15.6 \mathrm{~dB}$ according to Eq. 16 and will improve if determined against the SNR.

$E b / N_{0}=S I N A D\left[\frac{\Delta f}{\text { bitrate }}\right]$
4.6 Frequency Stability

Receive center frequency stability was checked by measuring the frequency where the best sensitivity is obtained and the center frequency did not shift with temperature either.

\section{Conclusion}

The averaged results on the communications performance of several VHF/UHF transceiver units for the CubeSats have been presented. The analysis began by first developing a simulation model to reason out the expected performance. The results are in accordance with the simulations and the analysis laid down. If the amplifier linearity is maintained, excellent radio performance may be expected. Appropriate thermal balance mechanism would avoid thermal memory effect problems and distortion during power conversion. To sum up, the work demonstrates robust performance of GMSK modulation and the transceiver impairments are small. The performance data may assist in system engineering activities such as the antenna design, spectrum analysis for the filing of bandwidth license, revising electric power budget and selecting the frequency for the highest quality line-of-sight link budget.

\section{Acknowledgment}

The authors would like to thank the contributors of the ZA-CUBE program, in particular, Axel Wottawa and Patrick Udhardt for automation of the thermal chamber and a host of other equipment, and Ben Dixon for the RF communications testset. We are indebted to Romain Le Gall for performing thermal IR testing and thermal FEA and Etnard Louw for testing several boards. We acknowledge Charl Jootse for many discussions and recognize ETSE Electronics for their role.

This work stems from the period when the principal investigator, Yaseen Zaidi, was a research fellow at the French South African Institute of Technology and was funded by the National Research Foundation (NRF) of South Africa.

\section{References}

1. Mars gets broadband connection, IEEE Spectrum, 15-15, February (2006)

2. Hwu, S.U., Loh, Y.C., Dobbins, J.A., Kroll Q.D., Sham, C.C.: Space shuttle uhf communications performance evaluation, IEEE A\&E Systems Magazine, 9-14, October (2005) 

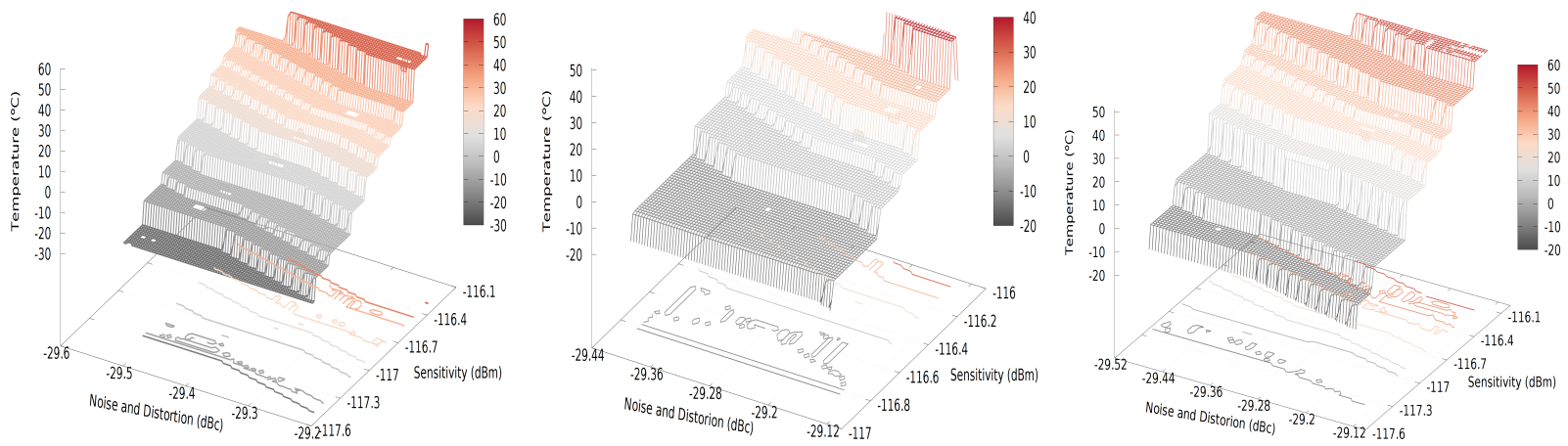

Fig. 23: SINAD vs. distortion at receiver frequency of $140 \mathrm{MHz}$ (left), $145 \mathrm{MHz}$ (middle) and $149 \mathrm{MHz}$ (right) during the thermal cycle

3. Bouwmeester J, Guo, J.: Survey of worldwide pico- and nanosatellite missions, distributions and subsystem technology, Acta Astronautica, 67, 854-862 (2010)

4. Joseph, A.T., Deshpande, M., O’Neill, P.E., Miles, L.: Development of vhf (240-270 MHz) antennas for soop (signal of opportunity) receiver for $6 \mathrm{u}$ cubesat platforms, Progress in Electromagnetic Research Symposium (PIERS), Shanghai, China, 8-11 August 2016

5. Sessler, G.M.A., Abelló, R., James, N., Maddè, R., Vassallo, N.: GMSK demodulator implementation for esa deep-space missions, Proceedings of the IEEE, 95, 11, 2132-2141 November (2007)

6. Shambayati, S., Lee, D.K.: GMSK modulation for deep space applications, IEEE Aerospace Conference, 2012

7. Anderson, J.B., Aulin, T., Sundberg, E.-C.: Digital phase modulation, Springer Science+Business Media, LLC, New York (1986)

8. Murota, K., Hirade, K: GMSK modulation for digital mobile radio telephony, IEEE Transactions on Communications, com-29, no. 7, 1044-1050 July (1981)

9. Simon, M.K: Bandwidth-efficient digital modulation with application to deep-space communications, Wiley (2001)

10. Pettai, R.: Noise in receiving systems, Wiley-Interscience, New York (1984)

11. Saleh, A.A.M.: Frequency-independent and frequencydependent nonlinear models of twt amplifiers, IEEE Transactions on Communications, com-29, 11, 1715-1720 November (1981)

12. Zaidi, Y., van Zyl, R.: A low cost testbed and testdesign methodology for nanosatellite sub-/systems, IEEE AFRICON, 2017

13. Josephson, G. C.: On the definition and measurement of occupied bandwidth, IEEE Transactions on Electromagnetic Compatibility, emc-12, 2, 33-37 (1970)

14. Fixed and base station fm receivers, National Institute of Justice, NIJ Standard-0206.01, U.S. Dept. of Justice, (1988) 